\title{
O SENSORIAMENTO REMOTO APLICADO NA ESTIMATIVA DA CONCENTRAÇÃO DE CLOROFILA NO MAR
}

\author{
JOĀO ANTONIO LORENZZETTI \\ Instituto de Pesquisas Espaciais - INPE \\ Säo Jose dos Campos, SP, Brasil
}

\section{SYNOPSIS}

In this paper the feasibility of estimating the chlorophyll content of ocean waters through the use of multispectral remote sensors on board of orbiting satellites, is discussed. Some aspects of the physical foundations of the interaction processes of visible light (380-770 nm) with the atmosphere and liquid mass and some models for this detection, are also discussed.

\section{Introdução}

Sem dúvida, é a fotossíntese um dos processos ambientais mais básicos e fundamentais para a vida na terra. No mar, ela é o começo de uma serie de eventos que liga a energia solar aos primeiros passos de uma longa cadeia alimentar que sustenta toda a fauna aquática. Assim, é quase desnecessário se ressaltar que, a variação temporal e espacial da produção fotossintética nos oceanos, e a sua relação com alguns parâmetros do próprio meio ambiente ou introduzidos pelo homem, merece um longo e exaustivo esforço de pesquisa, para que possa ser formada uma compreensão global da produção alimentar marinha.

Como se sabe, nos oceanos, o processo da fotossíntese é realizado por algas microscópicas, portadoras de clorofila denominadas fitoplancton. Sendo o pigmento de clorofila o principal agente no processo de transformação da energia solar em matéria orgânica, sua relação com a produtividade primária é evidente, e vários trabalho tém sido desenvolvidos para relacionar a concentração de clorofila com a produtividade. Ryther \& Yentsch (1957), p. ex., desenvolvem uma relação através da qual a produtividade orgânica, por unidade de área, pode ser calculada através do conhecimento da concentração de clorofila, a quantidade de radiação solar total que atinge a superfície do oceano e o coeficiente de extinção da luz visível na água.

Devido a restrições de numero de embarcações, equipamentos, pessoal especializado, etc., e tambem, sem dúvida, a dimensão das regiōes a serem estudadas $e$ as atuais velocidades dos navios oceanográficos, não é ainda possível amostrar o oceano com suficiente detalhamento espacial, repetitividade e sinopticidade desejada, Em muitos países, grandes esforços de capital e pessoal são investidos todos os anos, para se adensar a amostragem de parâmetros bioló gicos para o estudo de produtividade primária. A National Academy of.Sciences (1969) cita, que, cerca de US\$ 2.000.000,00 (ou atualmente bem mais) são gastos, por ano, na operação de navios envolvidos neste tipo de pesquisa.

Pressionada pelos problemas acima citados, nos últimos anos, com grande avanço de toda a tecnologia de sensores e o lançamento de inúmeros satélites artificiais dirigidos para recursos naturais, a possibilidade de se monitorar remotamente do espaço a concentração de clorofila nos oceanos, vem sendo estudada com afinco. $\mathrm{O}$ uso de sensores remotos orbitais, para o monitoramenteo de recursos marinhos, apresenta uma grande potencialidade, pois extensas regiões podem ser cobertas em apenas alguns minutos de sobrevôo, com grande repetitividade, conterindo assim, grande sinopticidade aos dados e permitindo um acompanhamento dinâmico dós fenômenos biológicos. Mapas periódicos, contendo a distribuição da concentração de clorofila nas camadas superficiais do mar, seriam de enorme valia para estudos de produtividade, e os mesmos, em conjunto com mapas contendo a distribuição da temperatura da superfície do mar, obtidos também remotamente do espaço, poderiam ser usados como indicadores de regiōes com alto potencial para a pesca. Um outro aspecto, também de grande interesse, seria o de detectar e estudar, por um sistema de sensor iremoto para clorofila, a dinâmica de florescência de algas, suas causas e conseqüências.

\section{A Deteção Remota da Concentração de Clorofila; Alguns Aspectos Teóricos}

O pressuposto fundamental, subjacente à técnica de deteção do teor de clorofila presente na água do mar, é que o espectro de radiância emergente do oceano, também chamado por espectro de cor, seja função da concentração de organismos clorofilados, embora possa ser, como realmente o é, função de inúmeros outros fatores. Uma mostra bastante clara, desta dependência demonstrada pelo es- pectro de radiância emergente do mar com a clorofila, pode ser encontrada no trabalho de Clarke et al. (1970) onde, ao compararmos os espectros obtidos na faixa do visivel (4000-7000 $\AA$ ), por um espec trometro instalado à bordo đe um aviāo, com dados de concentração de clorofila medidas in situ, verifica-se nitidamente uma sensível redução na porcentagem de luz azul quando a concentração de clorofila é aumentada de 0.1 para $3.0 \mathrm{mg}$ de clor. $/ \mathrm{m}$

Em última análise, a técnica de deteção remota da concentração de clorofila baseia-se numa adequada aquisição e tratamento dado a estes espectros de radiância emergente do mar, levando-se em conta as características particulares de interação da energia solar com os pigmentos clorofilados, com a própria água e demais componentes nela presentes, efeitos de interface ar-água e interferência atmosférica. Pode-se afirmar, com certeza, que o sucesso esperado na deteção remota da clorofila está intimamente vinculado a uma adequada formulação de um modelo matemático, não muito complexo para ser operacional, que relacione o espectro emergente, ou melhor ainda, a reflectância de irradiância, com a concentração de clorofila e que possibilite a eliminação ou minimização de tođas as outras componentes de radiância, que chegam ao sensor sem ter interagido diretamente com a massa liquida.

Designando por $\lambda$ o comprimento de onda da radiação medida, um sensor remoto situado a uma altitude $\mathrm{z}$ acima do nível do mar, e que esteja dirigido para o oceano abaixo, receberá, além da radiância $\mathrm{N}_{0}(\lambda)$ vinda da superfície do mar, e atenuada pela transmitância espectral atmosférica $T(\lambda)$, uma componente de radiância difusa $N^{*}(\lambda)$, chamada radiância de trajetória, que se origina por processo de retroespalhamento da energia solar na própria atmosfera. Assim, se $\mathrm{N}_{\mathrm{z}}(\lambda)$ é a radiância medida pelo sensor na altitude $\mathrm{z}$, temos

$$
N_{z}(\lambda)=N_{0}(\lambda) \times T(\lambda)+N^{*}(\lambda)
$$

A própria radiancia vinda da superfície do mar é constituída, em geral, por três componentes: $\mathrm{N}_{\mathrm{g}}(\lambda)$, uma parcela gerada por reflexão da luz direta do Sol em direçăo ao sensor e caracterizada visualmente por fortes brilhos intermitentes na superfície; $N_{\text {céu }}(\lambda)$, representando a radiância refletida pela luz difusa que incide sobre o mar e $\mathrm{N}_{\omega}(\lambda)$ a radiância vinda de dentro da massa líquida. Assim,

$$
N_{0}(\lambda)=N_{g}(\lambda)+N_{\text {céu }}(\lambda)+N_{\omega}(\lambda)
$$

E evidente que dessas três componentes, somente $N_{\omega}(\lambda)$ contém informação sobre os constituintes presentes na água e, levando-se em conta que $\mathrm{N}_{\mathrm{g}}(\lambda)$ pode ser várias ordens de grandeza maior que $\mathrm{N}_{\omega}(\lambda)$, todo cuidado deve ser empregado durante a aquisição dos dados remotos para que os padrões de brilho do Sol não estejam incluídos dentro do campo de visada do sensor.

Além de se ter que isolar $\mathrm{N}_{\omega}(\lambda)$ das demais componentes, não se deve esquecer que o seu módulo depende da quantidade de irradiância incidente sobre a superfície, e assim se torna necessário utilizar-se, em vez de $\mathrm{N}_{\omega}(\lambda)$, a reflectância de irradiância $\mathrm{R}(\lambda)$, definida como a razão entre a irradiância emergente do mar pela irradiância incidente, Vários são os trabalhos atualmente existente. sobre a conversão dos dados de radiância espectral coletada por um sensor orbital, em seus correspondentes valores de reflectância espectral. Pode-se destacar os trabalhos de Rogers et al (1974) e Gordon (1978), nos quais são desenvolvidos dois algorítmos simples de serem adaptados em computador e que realizam a conversão de $\mathrm{N}_{\mathbf{z}}(\lambda) \operatorname{em} \mathrm{R}(\lambda)$

Uma vez em condições de se obter, através de um sensor remoto multiespectral orbital, a reflectância de irradiância do mar, resta estabelecer-se um modelo que correlacione esta reflectância com o parâmetro biológico, concentração de clorofila, desejado. 


\section{A Relação entre as Propriedades Opticas e Biológicas da Água do Mar}

Uma pesquisa bibliográfica sobre este assunto revela a ausência de uma teoria amplamente reconhecida, que relacione as constantes óticas e biológicas de uma massa d'água. Mostrar-se-á, aqui um modelo desenvolvido por Smith \& Baker $(1978, a, b, c)$, que parte do pressuposto que os materiais suspensos e dissolvidos, presentes na água do mar, são de origem biológica em termos de concentração de pigmentos clorofilados.

Smith \& Baker (1978 a) introduzem o conceito de "estado bio-óptico" para representar uma medida do efeito total de processos biológicos sobre as propriedades ópticas de águas naturais. Várias são as razões apontadas pelos autores para a escolha do coeficiente de atenuação da irradiância difusa total $\mathrm{K}_{\mathrm{T}}(\lambda)$, como representativo deste estado bio-óptico. Em primeiro lugar, vários autores têm demonstrado a existência de uma forte correlação entre $\mathrm{K}_{\mathrm{T}}(\lambda)$ e a cencentração de clorofila. Gordon \& MacCluney (1975) mostraram que a máxima profundidade disponível, para ser estudada por sensoriamento remoto, é dada por $\mathrm{K} \cdot \mathrm{T}^{-1}$ Também, $\mathrm{K}_{\mathrm{T}}(\lambda)$ é o parâmetro que relaciona a irradiância propagando para baixo, numa profundidade $\mathrm{Z}, \mathrm{E}_{\mathrm{d}}(\mathrm{z}, \lambda)$, com a irradiância logo abaixo da superfície $E_{d}(0, \lambda)$ e sua medição em função da profundidade e comprimento de onda já não encontra grandes dificuldades técnicas.

O seguinte modelo linear é então apresentado, que relaciona as propriedades ópticas e biológicas da água do mar.

$$
\begin{aligned}
\mathrm{K}_{\mathrm{T}}(\lambda) & =\mathrm{K}_{\omega}(\lambda)+\mathrm{k}_{1}(\lambda) \cdot \mathrm{C}_{\mathrm{K}} & \left(\mathrm{C}_{\mathrm{K}}<1\right) \\
\mathrm{K}_{\mathrm{T}}(\lambda) & =\mathrm{K}_{\omega}(\lambda)+\mathrm{K}_{\mathrm{x} 2}(\lambda)+\mathrm{k}_{2}(\lambda) \cdot \mathrm{C}_{\mathrm{K}} & \left(\mathrm{C}_{\mathrm{K}}>1\right) \\
\text { com } \mathrm{k}_{1}(\lambda) & =\mathrm{K}_{\mathrm{x}^{2}}(\lambda)+\mathrm{k}_{2}(\lambda) & \left(\mathrm{C}_{\mathrm{K}}=1\right)
\end{aligned}
$$

onde

$$
C_{K}=\frac{1}{K_{T}^{-1}} \int_{0}^{K_{T}^{-1}} C(Z) d Z
$$

e a concentração média de clorofila numa coluna d'água até à profundidade $\mathrm{Z}=\mathrm{K}_{\mathrm{T}}^{-1}$, com $\mathrm{C}(\mathrm{Z})$ sendo a concentração de clorofila na profundidade $Z ; K_{\omega}(\lambda)\left[\mathrm{m}^{-1}\right.$ ] é o coeficiente de atenuação espectral difuso, parā uma água oceânica clara; $\mathrm{K}_{\mathrm{x}_{2}}(\lambda)\left[\mathrm{m}^{-1}\right]$ é uma variável que representa a contribuição média à atenuação espectral não diretamente atribuível à clorofila; $\mathrm{k}_{1}(\lambda)$ e $\left.\mathrm{k}_{2}(\lambda)\left[\mathrm{m}^{-1} \text { (mg pigmento } \mathrm{m}^{-3}\right)^{-1}\right]$ são os coeficientes de atenuaçẫo da irradiância espectral específicos da clorofila para águas com $C_{K}<1$ e $C_{K}>1$ respectivamente. Em Smith \& Baker (1978 b), tabela 1, p. 262 são apresentados os valores espectrais de todas estas constantes na gama de 350 a $700 \mathrm{~nm}$.

Tendo obtido $R(\lambda)$ remotamente e um modelo que relacione $C_{K}$ com $K_{T}(\lambda)$ necessita-se agora de um modelo que relacione direta ou indiretamente $K_{T}(\lambda) \operatorname{com} R(\lambda)$.

Morel \& Prieur (1977), partindo de um modelo desenvolvido por Gordon et al. (1975), chegam à seguinte relação

$$
R(\lambda)=0.33 \frac{b^{\prime}(\lambda)}{a(\lambda)}
$$

onde $b^{\prime}(\lambda)$ é o coeficiente de retroespalhamento $\left(\mathrm{m}^{-1}\right)$ e a $(\lambda)$, o coeficiente de absorção volumétrico. Nesta expressão tem-se a $(\lambda)$, que não havia aparecido até então; entretanto, Preisendorfer (1976), apresenta a seguinte relação, que permite a eliminação de a $(\lambda)$

$$
a(\lambda) \cong 0.75 \quad \frac{K_{\mathrm{T}}(\lambda)}{1+2 \mathrm{R}(\lambda)}
$$

Substituindo (6) em (5) vem:

$$
R(\lambda)=\frac{b^{\prime}(\lambda)}{2.27 K_{T}(\lambda)-2.0 b^{\prime}(\lambda)}
$$

Chega-se então, através das equações (7) e (3), a uma relação funcional entre $R(\lambda)$ e $C_{K}$, a menos do coeficiente de retroespalhamento $b^{\prime}(\lambda)$, constituído pela soma de um retroespalhamento molecular, $\sigma^{\prime} \omega(\lambda)$, determinado por Morel (1974), e um retroespalhamento por partículas b' ${ }_{P}(\lambda)$.

\section{Duas Abordagens com boas Perspectivas de Sucesso}

Seguindo um esquema proposto por Jain \& Miller (1976), uma maneira de se solucionar a equação (7) para $C_{K}$, seria usar-se um esquema de optimização qualquer que minimize uma função objetivo consistindo de uma soma ponderada das diferenças percentuais entre as reflectâncias espectrais calculadas por (7) e medidas pelo sensor em comprimentos de onda $\lambda_{i}$ escolhidos.

Por geração de números aleatórios seriam gerados os parâmetros $C_{K}$ e b' $\left(\lambda_{j}\right)$ iniciais que, usados nas equações (3) e (7), forneceriam as reflectâncias calculadas $R_{c}\left(\lambda_{i}\right)$. A função objetivo $F$ a ser minimizada seria dada por

$$
F=\sum_{i=1}^{n} \frac{R_{c}\left(\lambda_{i}\right)-R_{\text {medida }}^{\left(\lambda_{j}\right)}}{R_{c}\left(\lambda_{i}\right)}
$$

onde $\mathrm{n}$ representa o numero de canais do sistema sensor.

Por um processo de busca univariada, o programa de computador iria então a cada passo variando estes valores de entrada até que o mínimo global em $F$ fosse obtido. As variáveis finais $C_{K}$ e b'. que forneçam este minimo em $\mathrm{F}$, devem ser aquelas buscadas.

\section{b) Abordagem de Wilson-Austin-Smith}

Traba!thando com o modelo de Smith \& Baker (1978c) para a reflectância de irradiância em três comprimentos de onda $\lambda_{1}, \lambda_{2}, \lambda_{3}$ distintos, Wilson et al. (1978) chegam a seguinte expressão

$$
C_{K}=\begin{array}{lll}
a_{1}+a_{2} \quad & R\left(\lambda_{3}\right)+a_{3} \\
& \left.R\left(\lambda_{2}\right)+\lambda_{1}\right) \\
& R\left(\lambda_{2}\right) \\
& R\left(\lambda_{3}\right)+a_{6}+a_{5}\left(\lambda_{1}\right) \\
& R\left(\lambda_{2}\right) \\
& R\left(\lambda_{2}\right)
\end{array}
$$

onde as constantes $a_{i}(i=1, \ldots, 6)$ devem ser calculadas, para cada região, por técnicas de mínimos quadrados, através da análise dos dados de clorofila medidos in situ e os correspondentes $R\left(\lambda_{i}\right)$. Wilson, op. cit., ao aplicarem este modelo para dados obtidos por um sensor a bordo de avião, para a região da Baía de New York, chegam a um coeficiente de correlação superior à 0.90 entre os dados de clorofila medidos e calculados.

\section{Perspectivas Futuras}

Como mostra Yentsch (1960), o espectro de absorção da clorofila possui dois picos localizados em 445 e $675 \mathrm{~nm}$ aproximadamente, e uma região de mínima absorção por volta de $550 \mathrm{~nm}$. Assim, todo sistema sensor remoto, para a deteção de clorofila, deveria coletar dados de irradiância espectral ao menos nestes comprimentos de onda (ou próximo deles), uma vez que a relação entre as reflectâncias nestes canais serviria para realçar as variaçōes no espectro de luz emergente do mar, devidas à variação de concentração de clorofila.

A partir de novembro de 1978, com o lançamento do satélite NIMBUS G da NOAA (National Oceanic and Atmospheric Administratión) será pela primeira vez possível se obter dados de um sistema sensor orbital especialmente construido para a deteção de clorofila e que satisfaz as especificaçōes acima descritas. Este sistema, chamado Coastal Zone Color Scanner (CZCS) é um radiômetro de varredura com seis canais assim constituídos:

$\begin{array}{cc}\text { Canal } & \text { Faixa espectral }(\mathrm{nm}) \\ 1 & 433-453 \\ 2 & 510-530 \\ 3 & 540-560 \\ 4 & 660-680 \\ 5 & 700-800 \\ 6 & 10.5-12.5(\mathrm{jm})\end{array}$

Como se vê, os quatro primeiros canais, optimizados com alto ganho, poderão fornecer dados de altíssima qualidade para a deteção de clorofila. O canal 5 , em baixo ganho, é equivalente ao canal 6 do sistema MSS do satélite LANDSAT e foi incluído para servir de intercomparação com este outro sistema. O canal 6 fornecerá dados sobre o campo de temperatura superficial do mar. 
Tudo indica que os dados fornecidos por este sistema, processados adequadamente para gerar $R\left(\lambda_{i}\right)$ e usados em modelos como aqueles apresentados acima, poderão fornecer boas estimativas dos campos de clorofila superficiais do mar. Deve-se entretanto, ressaltar a necessidade de um trabalho de coleta de dados de clorofila in situ que servirão, ou para ajustar as constantes dos modelos, ou para aferir os resultados. Assim, um trabalho de equipe, envolvendo pesquisadores da área de sensoriamento remoto e oceanógrafos biólogos será extremamente proveitoso para ambos os setores.

\section{Bibliografia}

CLARKE, G. E. ; EWING, G. C. \& LORENZEN, C. J. 1970. Spectra of backscattered light from the sea obtained from aircraft as a measure of chlorophyll concentration. Science, 167: 1119-1121.

GORDON, H. R. 1978. Removal of atmospheric effects from satellite imagery of the oceans. Appl. Opt., $17(10): 1631-1636$.

- . - \& MacCLUNEY, W. R. 1975. Estimation of the depth of sunlight penetration in the sea for remote sensing. Appl. Opt., 14 (2): 413-416.

JAIN, S. C. \& MILLER, J. R. 1976. Subsurface water parameters optimization approach to their determination from remotely sensed water color data. Appl. Opt., 15 (4): 886-890.

MOREL, A. 1974. Optical properties of pure water pure sea water. In: Jerlov, N. G. \& Steemann-Nielsen, E., eds. - Optical aspects of oceanography. London, Academic Press, p. 4-24.
\& PRIEUR, L. 1977. Analysis of variations in ocean color. Limnol. Oceanogr.. 22 (4): 709-722.

NATIONAL ACADEMY OF SCIENCES. 1969. Spectra of the light backscattered from the upper ocean. In: Useful application of.Earth-oriented satellites, p. 44

PREISENDORFER, R. W. 1976. Hydrologic optics. U. S. Department of Commerce, NOAA, Environmental Research Laboratories, Honolulu, Hawaii.

ROGERS, R. H., ; PEACOCK, K, \& SHAN, N. J. 1974. A technique for correcting ERTS data for solar atmospheric effects. Third Earth Resources Technology Symposium, p. 1787 . -1802 .

RYTHER, J. H. \& YENTSCH, C. S. 1957. The estimation of phytoplankton production in ocean from chlorophyll and light data. Limnol Oceanog., 2:281-286.

SMITH, R. C. \& BAKER, K. S. 1978a. The bio-optical state of ocean waters and remote sensing. Limnol. Oceanogr., 23(2): 247-259.

- - - \& \&-_- 1978b. Optical classification of natural waters. Limnol. Oceanogr., 23(2): 260-267. - \& $-\ldots-1978$ c. The remote sensing of chlorophyll. XXth Meeting COSPAR Symposium on the Contribution of Space Observations to Global Food Information Systems.

WILSON, W. H., AUSTIN, R. W. \& SMITH, R. C. 1978. Optical remote sensing of chlorophyll in ocean waters. Preprint of the Proceedings of the Twelfth International Symposium on Remote Sensing of the Envifonment, Manila.

YENTSCH, C. S. 1960. The influence of phytoplankton pigments on the color of the sea. Deep sea Res., 7:1-19. 